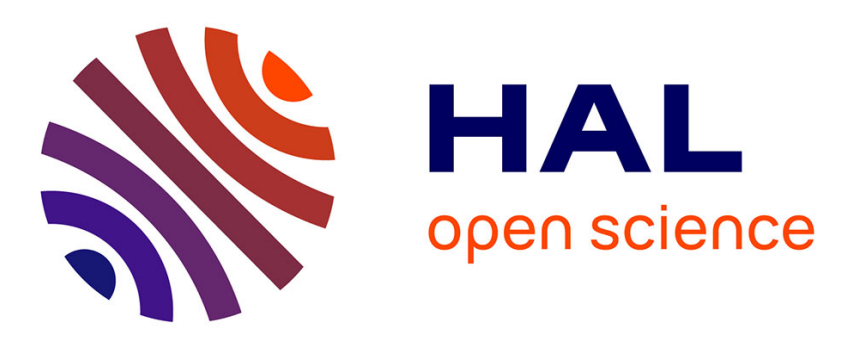

\title{
Influence of pores on crack initiation in monotonic tensile and cyclic loadings in lost foam casting A319 alloy by using $3 \mathrm{D}$ in-situ analysis
}

Long Wang, Nathalie Limodin, Ahmed El Bartali, Jean-Francois Witz, Rian Seghir, Jean-Yves Buffiere, Eric Charkaluk

\section{To cite this version:}

Long Wang, Nathalie Limodin, Ahmed El Bartali, Jean-Francois Witz, Rian Seghir, et al.. Influence of pores on crack initiation in monotonic tensile and cyclic loadings in lost foam casting A319 alloy by using 3D in-situ analysis. Materials Science and Engineering, 2016, 673, pp.362-372. 10.1016/j.msea.2016.07.036 . hal-01433827

\section{HAL Id: hal-01433827 https://hal.science/hal-01433827}

Submitted on 21 Oct 2019

HAL is a multi-disciplinary open access archive for the deposit and dissemination of scientific research documents, whether they are published or not. The documents may come from teaching and research institutions in France or abroad, or from public or private research centers.
L'archive ouverte pluridisciplinaire HAL, est destinée au dépôt et à la diffusion de documents scientifiques de niveau recherche, publiés ou non, émanant des établissements d'enseignement et de recherche français ou étrangers, des laboratoires publics ou privés. 


\title{
Influence of pores on crack initiation in monotonic tensile and cyclic loadings in lost foam casting A319 alloy by using 3D in-situ analysis
}

\author{
Long WANG $^{1 *}$, Nathalie LIMODIN ${ }^{1}$, Ahmed EL BARTALI ${ }^{1}$, Jean-François WITZ ${ }^{1}$, Rian SEGHIR ${ }^{1}$, \\ Jean-Yves BUFFIERE ${ }^{2}$, Eric CHARKALUK ${ }^{1}$ \\ 1. Univ. Lille, CNRS, Centrale Lille, Arts et Metiers Paris tech, FRE 3723 - LML - Laboratoire de Mecanique de \\ Lille, F-59000 Lille, France \\ 2. Laboratoire Matériaux, Ingénierie et Sciences (MATEIS), CNRS UMR5510, INSA-Lyon, 20 Av. \\ Albert Einstein, 69621 Villeurbanne, France
}

\begin{abstract}
Lost Foam Casting (LFC) process is replacing the conventional gravity Die Casting (DC) process in automotive industry for the purpose of geometry optimization, cost reduction and consumption control. However, due to lower cooling rate, LFC produces in a coarser microstructure that reduces fatigue life. In order to study the influence of the casting microstructure of LFC Al-Si alloy on damage micromechanisms under monotonic tensile loading and Low Cycle Fatigue (LCF) at room temperature, an experimental protocol based on the three dimensional (3D) in-situ analysis has been set up and validated. This paper focuses on the influence of pores on crack initiation in monotonic and cyclic tensile loadings. X-ray Computed Tomography (CT) allowed the microstructure of material being characterized in 3D and damage evolution being followed in-situ also in 3D. Experimental and numerical mechanical fields were obtained by using Digital Volume Correlation (DVC) technique and Finite Element Method (FEM) simulation respectively. Pores were shown to have an important influence on strain localization as large pores generate enough strain localization zones for crack initiation both in monotonic tensile and cyclic loadings.
\end{abstract}

Keywords: tomography, finite element method, aluminium alloys, casting, fatigue

${ }^{*}$ Corresponding author. Current contacts and address: Tel. +86 108853 1403; E-mail address: longwang_calt@163.com; Science and Technology on Reliability and Environment Engineering Laboratory, Beijing Institute of Structure and Environment Engineering, Beijing 100076, China 


\section{Introduction}

Thanks to high strength to weight ratio, good machinability, corrosion resistance, optimum surface finish and high electrical and thermal conductivity, aluminum alloys are widely used in automotive industry ${ }^{[1,2]}$. The Al-Si alloy is one of the most commonly used $\mathrm{Al}$ alloys in automotive industry mainly in engine parts. Many alloying elements, such as $\mathrm{Cu}, \mathrm{Ni}, \mathrm{Mg}$, may be added to improve its performances. During the casting process, defects are inevitably produced. Therefore the complex microstructure of Al-Si alloys inherited from the casting process affects the mechanical behavior ${ }^{[3,4]}$.

Cylinder heads in Al-Si alloy are submitted to severe thermomechanical stresses (temperature gradient, high cyclic loadings, and thermal shock) with temperature variation from 20 to $250^{\circ} \mathrm{C}$ ${ }^{[5]}$. In particular, the inter-valve zone in the fire deck is the most critical area. In order to resist to these in-service conditions, the material should have good mechanical properties especially in Low Cycle Fatigue (LCF).

The conventional casting process used to manufacture cylinder head is gravity Die Casting (DC) process ${ }^{[6]}$, but it is progressively being replaced by the Lost Foam Casting (LFC) process for the purpose of geometry optimization, cost reduction and consumption control ${ }^{[7]}$. However, the microstructure is not only influenced by the chemical composition of the investigated alloy but also by the cooling rate ${ }^{[8,9]}$ which is much lower for LFC, around $0.8^{\circ} \mathrm{C} / \mathrm{s}$, than for DC, around $30^{\circ} \mathrm{C} / \mathrm{s}^{[10]}$. Secondary Dendrite Arm Spacing (SDAS) is a microstructural parameter which reflects the coarseness of the microstructure: it is about $35 \mu \mathrm{m}$ in a DC A356 alloy, while it increases to $85 \mu \mathrm{m}$ in a LFC A356 alloy ${ }^{[10]}$. Besides, with a lower cooling rate, the size and volume fraction of pores and the size of the intermetallic particles and eutectic silicon increase, and the shape of the eutectic silicon become more acicular ${ }^{[11,12,13]}$. Thus, the Al-Si alloys cast by the LFC process present coarser microstructure and large pore volume fraction.

Casting microstructure resulting from this process has a major influence on the fatigue properties as it has been observed that the fatigue life of LFC A319 alloy is drastically reduced compared to DC A319 alloy while the strain-stress hysteresis loop is virtually unchanged ${ }^{[10]}$. Moreover, in past years, some studies have helped to reveal the damage mechanisms of $\mathrm{Al}-\mathrm{Si}$ alloys in High Cycle Fatigue (HCF). Fatigue crack initiation occurs preferentially at various microstructural inhomogeneities ${ }^{[14]}$. For example, above a critical size, pores play a decisive role by providing preferential crack initiation sites ${ }^{[3,15,16]}$. In addition, they accelerate fatigue crack propagation as they increase the stress level ${ }^{[3]}$. Pores at the surface ${ }^{[17]}$ or near the specimen surface ${ }^{[18]}$ are reported as the most dangerous defects. They have the greatest propensity for acting as crack initiation sites and for decreasing the fatigue life as they create regions of high stress concentrations. The porosity level was found to be a first order parameter which greatly alters both the average number of cycles to failure and the lifetime scatter, especially at high stress level ${ }^{[19]}$ As 
we know, a pore with small radii curvature at their surfaces induce larger stress concentration, thus the pores shape could also influence the mechanical behavior ${ }^{[20]}$. However, according to [14], shape may have a negligible effect on fatigue crack initiation compared to other significant factors such as size.

While most of these studies were performed in High Cycle Fatigue (HCF) regime, only a few focused on the damage mechanisms in Al-Si alloy with large casting defects in Low Cycle Fatigue (LCF) regime ${ }^{[10,21]}$. Moreover, the previous studies ${ }^{[10,21]}$ were not performed in-situ, even if post-failure analysis makes it possible to track the defect at the initiation site, it does not allow studying the relationship between microstructure and crack growth kinetic in the bulk. In LCF, this analysis is made even more difficult as failure often results from multicracking rather than from a single crack ${ }^{[21]}$.

The aim of the present study is to understand the damage micromechanisms of LFC Al-Si alloy, in particular crack initiation, under both monotonic tension and LCF tests at room temperature. At first the microstructure of the studied material was characterized thoroughly both on surface and in the bulk. Mechanical tests with 3D in-situ observations were then performed to follow crack initiation and propagation in the bulk. The 3D images obtained were analyzed using the Digital Volume Correlation (DVC) technique to study the relation between the measured mechanical field and the observed damage localization. Finite Element Method (FEM) simulation using 3D mesh models based on pores and solid matrix was used to assess the influence of pores on strain distribution in order to better understand the damage mechanisms.

\section{Experimental and numerical methods}

\subsection{Material and casting process}

Sample used in this study have been extracted from an A319 LFC cylinder head which had not undergone post casting heat treatment. The chemical composition is given in Table 1. LFC consists in using a polystyrene assembly as a pattern, the pattern is dipped into a ceramic refractory coating then placed into sand; it is vaporized when the melt is poured. As stated in introduction, the microstructure is influenced by the chemical composition of the investigated alloy and by the cooling rate during casting process.

\begin{tabular}{|c|c|c|c|c|c|c|c|c|c|c|c|}
\hline Si & Fe & Cu & Mn & $\mathrm{Mg}$ & $\mathrm{Ni}$ & $\mathrm{Zn}$ & $\mathrm{Pb}$ & $\mathrm{Sn}$ & $\mathrm{Ti}$ & \multicolumn{2}{|c|}{$\mathrm{Zr}, \mathrm{V}, \mathrm{P}, \mathrm{Sr}$} \\
\cline { 3 - 9 } & & & & & & & & & & each & total \\
\hline $\mathbf{5 . 3 - 7 . 5}$ & $\leqq \mathbf{0 . 6}$ & $\mathbf{3 - 3 . 8 0}$ & $\leqq 0.50$ & $0.25-0.40$ & $\leqq 0.30$ & $\leqq 0.40$ & $\leqq 0.10$ & $\leqq 0.05$ & $\leqq 0.20$ & $\leqq 0.03$ & $\leqq 0.1$ \\
\hline
\end{tabular}

Table 1 Chemical composition of A319 (AlSi7Cu3) LFC alloy (wt.\%) 


\subsection{Microstructure characterization}

The coarser microstructure of A319 LFC alloy used in this study was revealed using Scanning Electron Microscope (SEM) as displayed in Figure 1(a). Besides Al matrix, the most important microstructure constituents are pores and hard inclusions, i.e. eutectic $\mathrm{Si}$, iron intermetallics, and copper containing phases. Pores have coral-like (shrinkage cavities) appearance or approximate spherical shapes (gas pores). The Al matrix occupies the largest surface area and presents dendritic morphology. The eutectic silicon particles are acicular branched plates. The iron intermetallics present a "Chinese script" morphology or a needle/plate-like morphology. The copper containing phases present different complex morphologies.
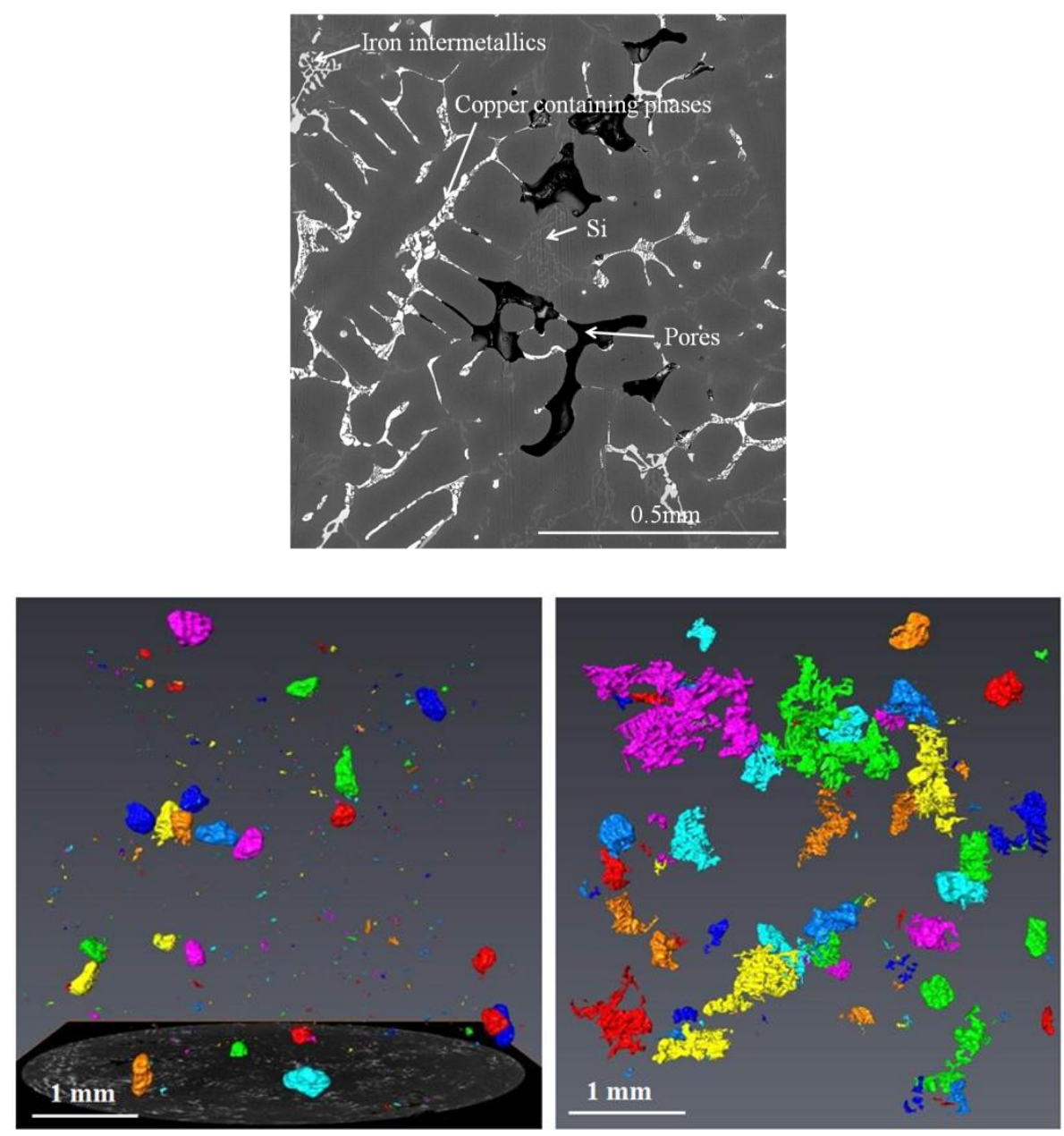

Figure 1 (a) BSE image of microstructure of LFC A319 alloy; 3D rendering of pores in LFC A319 alloy: (b) gas pores and (c) shrinkage pores

X-ray Computed Tomography (CT), which allows the non destructive 3D characterization of microstructural constituents of a material is now an established technique in material science and can also be sued to study fatigue behavior of metals ${ }^{[22,23,24]}$. The pores, on which we focus in this paper, were characterized in 3D using laboratory tomography (Lab-CT) with a voxel size of $3 \mu \mathrm{m}$ in a large volume of material (about $70 \mathrm{~mm}^{3}$ ). Figures $1(\mathrm{~b})$ and (c) show 3D renderings 
of two types of pores: small size gas pores and large size shrinkage pores. These 2 types of pores are distinguished by using a 'sphericity (F)' parameter which corresponds to the ratio between the object volume and the volume of a sphere having the same surface as the object, i.e. $F=6 \sqrt{\pi} \frac{\text { volume }}{\sqrt{\text { surface }^{3}}}$ with $\mathrm{F}=1$ for an ideal sphere. In Figures 1 (b) and (c), pores with sphericity (F) more than 0.4 are identified as gas pores while pores with sphericity (F) less than 0.4 are identified as shrinkage pores. Gas pores resulting from entrapped gases are rounded individual objects; shrinkage pores forming during the cooling stage are tortuous individual object. The volume fraction of pores in the studied material is about $1 \%$. The Feret diameter, which is defined as the maximum distance between two parallel planes restricting the object perpendicular to that direction, is used to characterize the size of pores in 3D. Compared with the commonly used 'Equivalent diameter', which is the diameter of a sphere that would have the same volume as the object, the Feret diameter is able to characterize not only the volume of a pore, but also its shape, which is very important for the mechanical behavior. The maximum Feret diameter of the pore we assumed here exceeds $1.5 \mathrm{~mm}$.

\subsection{Mechanical tests}

\subsubsection{Specimens}

The developed experimental protocol in this paper consists in using X-ray tomography for the in-situ observations during mechanical tests. However, for a given detector, which results in a specific size of image in pixels, a larger scan volume yields a lower resolution. Thus only small size specimens (Figure 2) are suitable for in-situ observations using X-ray tomography at a resolution sufficient to capture cracks (voxel size in the micro range).

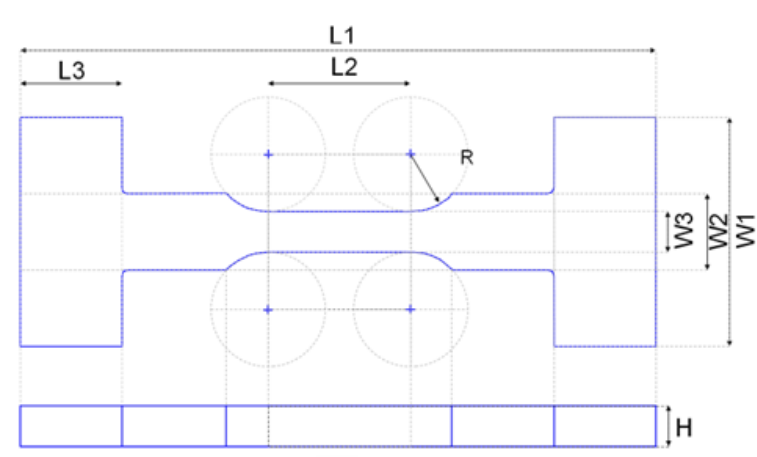

\begin{tabular}{|c|c|c|}
\hline \multirow{2}{*}{ Parameters } & \multicolumn{2}{|c|}{ length $(\mathrm{mm})$} \\
\cline { 2 - 3 } & $\begin{array}{c}\text { tensile } \\
\text { tests }\end{array}$ & $\begin{array}{c}\text { fatigue } \\
\text { tests }\end{array}$ \\
\hline L1 & 25 & 24 \\
\hline L2 & 4.5 & 4 \\
\hline L3 & 4 & 5 \\
\hline W1 & 9 & 12 \\
\hline W2 & 3 & 4 \\
\hline W3 & 1.6 & 2.6 \\
\hline H & 1.6 & 2.6 \\
\hline R & 0.7 & 2 \\
\hline
\end{tabular}

Figure 2 Size of specimens

Specimens extracted by electro discharge machining from automotive cylinder heads provided by the PSA Company. They were cut from or close to the fire deck area. Due to different cooling rates, the coarseness of the microstructure, which is reflected by SDAS, will vary in different parts of a cylinder head. Thus, the SDAS of the specimens taken outside of the fire deck was measured to ensure that they are indeed representative for the coarseness of the microstructure in the fire deck with a SDAS of about $74 \mu \mathrm{m}{ }^{[10]}$. The specimens which have a 
square cross section were mechanically polished on both faces down to a $1 / 4 \mu \mathrm{m}$ surface finish to avoid crack initiation from machining scratches and to allow for post-mortem analysis using SEM ${ }^{[25]}$. The edges of the corner were slightly polished using abrasive paper by hand in order to avoid the stress concentration.

A preliminary 3D characterization using Lab-CT (MATEIS laboratory, Lyon, France) was performed on the extracted specimens at about $5 \mu \mathrm{m}$ voxel size; these medium resolution images allow revealing the size and shape of the large pores in the bulk of the specimens gauge length. The purpose of such a characterization is threefold:

(1) The most suitable specimens were selected for the following tests. This represents about one fourth of the extracted specimens. The chosen specimens have no large defects near the shoulders while they have defects with a size compatible with the specimen cross-section in the gauge length.

(2) The Region of Interest (ROI) where the in-situ observations were performed with a higher resolution, i.e. at a voxel size of 1.695 (resp.1.625) $\mu \mathrm{m}$ for tensile (resp. fatigue) tests, but in a smaller volume, i.e. with a scan height of 1.7 (resp. 3.5) $\mathrm{mm}$ vs. gauge length of 4.5 (resp. 4) $\mathrm{mm}$ for tensile (resp. fatigue) tests, was selected. The selected areas contain a cluster of pores.

(3) In order to ensure that the small size specimens could represent the studied material, the pores in the small tensile/fatigue specimens were characterized quantitatively in $3 \mathrm{D}$ and compared with that in a larger volume of material.

\subsubsection{Tensile tests}

In situ tensile tests characterized by Lab-CT were performed (MATEIS Lab.) tensile tests with in-situ 3D observation. Although Lab-CT does not allow distinguishing eutectic Si from Al matrix, it allows studying the influence of pores on damage mechanisms and it is easier to access than SR-CT. An $80 \mathrm{kV}$ acceleration voltage was selected to enable for at least $10 \%$ transmission of incoming X-ray beam. A set of 900 radiographs was recorded while the specimen was rotating over $360^{\circ}$ along its vertical axis. An acquisition time per image of $500 \mathrm{~ms}$ yielded a scan duration of $45 \mathrm{~min}$. An in-situ test rig ${ }^{[26]}$ was installed in the tomography chamber in order to load the specimen (Figure 3 (a)). The specimen was loaded step by step and a scan was performed at each step with a $1.695 \mu \mathrm{m}$ voxel size (Figure 3 (b)). Six loading steps were applied: the corresponding stresses are 147, 165, 176, 188, 192 and, 198 MPa respectively. The test ended at the $6^{\text {th }}$ step after a number of cracks had been observed in the tomography images. 
(a)
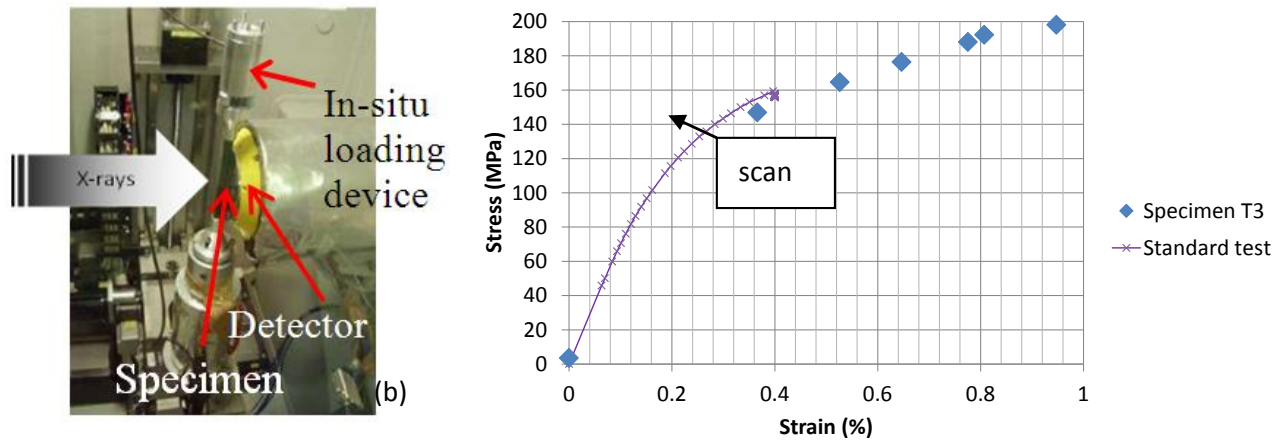

Figure 3 (a) in-situ tensile test rig inside the tomography chamber; (b) comparison of stress-strain curves from DVC and from a standard test on A319 alloy (each point corresponds to a scan acquisition while test was stopped and the specimen was held under load)

\subsubsection{Fatigue tests}

The same experimental protocol as for tensile tests was applied on fatigue tests with in-situ observation by synchrotron tomography performed on TOMCAT beamline at the Swiss Light Source (SLS) in Villigen, Swiss. With an exposure time of $300 \mathrm{~ms}$ per image, one scan lasted about $7 \mathrm{~min}$. Compared with Lab-CT, the scan duration of SR-CT is more compatible with fatigue testing. In addition, thanks to the phase contrast provided by SR-CT, eutectic Si, which cannot be distinguished from Al matrix using Lab-CT, was revealed. A specially designed fatigue machine ${ }^{[27]}$ was used to load the specimen in-situ. The fatigue test (Maximum stress = $130 \mathrm{MPa}$; Stress ratio $\approx 0.1$; Cyclic frequency: $0.3 \sim 10 \mathrm{~Hz}$ ) was interrupted periodically for scan acquisition; only a few cycles were between each acquisition growth. 3D images, with a voxel size of $1.625 \mu \mathrm{m}$, were acquired at minimum load before fatigue cycling and at both minimum and maximum loads of each studied cycle during the fatigue experiment.

\subsection{Experimental and numerical mechanical fields}

\subsubsection{Experimental fields}

The experimental displacement fields were measured using the DVC technique, which is an extension of the well-developed Digital Image Correlation (DIC) method; one can refer to reference ${ }^{[28]}$ for its principle. DVC measurements were performed either using the Mechanical Image Correlation (MIC3D) algorithm developed by J. Réthoré (LaMCoS, France) for tensile tests, and YaDICs - Digital Image Correlation 2D/3D software ${ }^{[29]}$, which is optimized for treating large data set in a limited time, for fatigue tests. The parameters of DVC measurements are shown in Table 2. 


\begin{tabular}{|c|c|c|}
\hline Test type & Tensile tests & Fatigue test \\
\hline \multirow{2}{*}{ Image size } & $800 \times 820 \times 1000$ voxels & $1450 \times 1560 \times 2160$ voxels \\
& $\left(1.36 \times 1.39 \times 1.36 \mathrm{~mm}^{3}\right)$ & $\left(2.36 \times 2.54 \times 3.51 \mathrm{~mm}^{3}\right)$ \\
\hline \multirow{2}{*}{ ROI } & $688 \times 768 \times 768$ voxels & $1392 \times 1320 \times 800$ voxels \\
& $\left(1.17 \times 1.3 \times 1.3 \mathrm{~mm}^{3}\right)$ & $\left(2.26 \times 2.14 \times 1.3 \mathrm{~mm}^{3}\right)$ \\
\hline Element size & 16 voxel $(27.2 \mu \mathrm{m})$ & 8 voxels $(13.0 \mu \mathrm{m})$ \\
\hline DVC algorithm & MIC3D & YADICS \\
\hline
\end{tabular}

Table 2 Parameters of DVC measurements

The displacement field measurement uncertainty ${ }^{[30]}$ was calculated in order to assess the feasibility of DVC for A319 alloy. For Lab-CT, two scans were taken: one at a reference position and another one after a translation along the beam direction. As the beam of lab-CT is conical, the voxel size is changed (from 1.7 to $1.695 \mu \mathrm{m}$ in the present case). Thus a known displacement field in all directions, i.e. a dilatation, which corresponds to a maximum displacement of 1.5 voxel, was applied ${ }^{[30]}$. For SR-CT, after taking a reference scan, another one was trecorded after a small displacement (10 $\mu \mathrm{m}$ in the present case) of the specimen in a direction perpendicular to the beam direction and to the axis of rotation of the stage. The measurement accuracies in this study (Figure 3(c)) are acceptable as the uncertainty remains subvoxel even for the smallest size of element investigated for both SR-CT and Lab-CT. The measurement accuracy strongly depends on the number of natural markers. Eutectic Si, which cannot be revealed using Lab-CT, was revealed using SR-CT, thus SR-CT reveals more markers and has a higher measurement accuracy. Measurement uncertainty always depends on the element size: It decreases when element size incrases, but a large element size reduces the spatial resolution. As a compromise between measurement accuracy, (i.e. uncertainty), and spatial resolution (i.e. element size), an appropriate element size, was selected for each measurement: for Lab-CT, the selected element size is $27.2 \mu \mathrm{m}$ (16 voxels) (corresponding uncertainty is $0.370 \mu \mathrm{m}(0.176$ voxels $))$, for SR-CT, the selected element size is $13.0 \mu \mathrm{m}$ (8 voxels) (corresponding uncertainty is $0.112 \mu \mathrm{m}(0.069$ voxels $)$ ).

For the tensile test, correlation was performed between the reference image (undeformed state) and each deformed image, the displacement and strain fields at each load step were obtained. As only the applied load was known through the in-situ test rig, the longitudinal strain field $\left(\varepsilon_{\mathrm{zz}}\right)$ measured from DVC was averaged over the whole volume at each loading step in order to determine the stress-strain curve named "in-situ tensile test" in Figure 3 (b) Compared with the curve of the same material obtained from a conventional test, i.e. with appropriate extensometer measurement on larger specimens, the curve derived from DVC can be considered representative. The small dispersion may be due to the dispersion of microstructure, i.e. size and volumetric fraction of pores. This confirms the validity of the DVC technique to analyze the tensile test.

For the fatigue tests, the DVC measurements were performed between the minimum loading of 
$1^{\text {st }}$ cycle and the maximum loading of the following cycles. The 3D field of the cumulative equivalent von Mises strain could thus be obtained. The mean value of the deformation along the loading direction, which cannot be measured in-situ during the fatigue tests, was also obtained from DVC measurements.

\subsubsection{Numerical mechanical fields}

To check if the strain distribution due to the pores coincides with the measured strain field, FEM simulation was performed. The FEM mesh model representing the solid matrix and the pores was obtained from the 3D tomographic image using Avizo software ${ }^{[31]}$ (Figure 4). The mesh process can be summarized as the following five steps:

- Segmentation: A region growing method is used for the segmentation of the solid matrix, i.e. aluminium matrix with hard inclusions included. It allows obtaining a 3D model of the specimen and of all the pores.

- Surface generation: Avizo uses a marching cubes algorithm to generate a triangular approximation of the $3 \mathrm{D}$ surface that delimitates the thresholded area. The number of triangles is about $3 \times 10^{6}$ at this step (Figure 4 (a)).

- Simplification of the 3D surface: To decrease the number of triangles, a simplification can be done by imposing the minimum and maximum sizes of the triangles to 0.2 and 10 voxels respectively. The number of triangles is reduced to less than $3 \times 10^{5}$.

- Remeshing of the surface: As the size of the element is still too small, two steps of remeshing are performed to decrease the number of triangles by globally coarsening the triangles while maintaining locally a refined mesh at the regions of high curvature, i.e. at pores and specimens corners. At each step, the number of triangles is reduced by $50 \%$ using the 'best isotropic vertex placement' option then the 'high regularity' option which allows regularizing the simplified mesh (Figure 4 (b)).

- Volumetric grid: All these operations may introduce errors, i.e. intersections, or distortions in the triangles that need to be corrected in a semi-automatic way in Avizo. Then a tetrahedral grid can be generated to fill the volume enclosed by the 3D surface mesh. The mean length of the inner tetrahedra is about the same as that of surface triangles, i.e. about 10 voxels, and the final number of tetrahedron is about $2 \sim 3$ millions (Figure 4 (c)). 


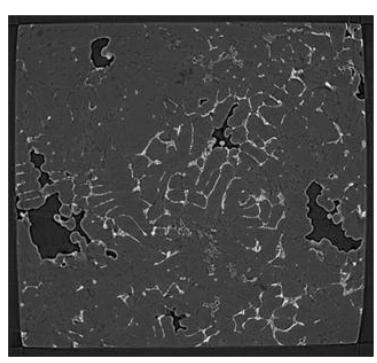

Tomographic image

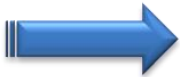

Surface Simplification Manual surface edition Remesh...

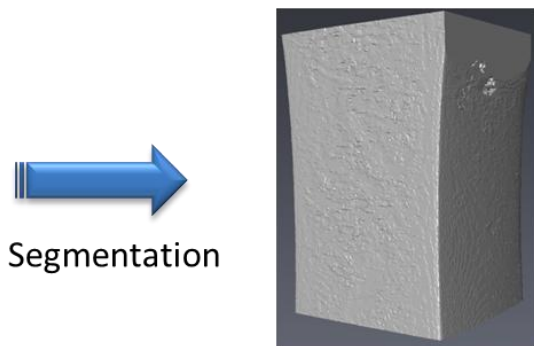

Solid matrix surface

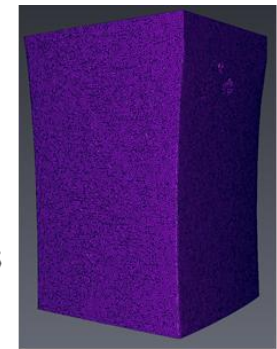

Triangular surface

Mesh on surfaces

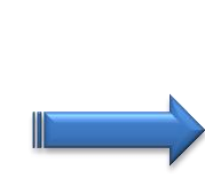

FE simulation

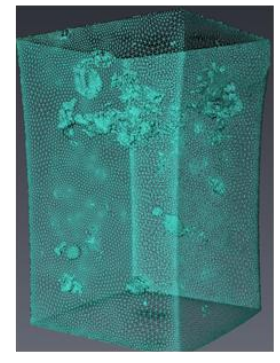

Volumetric tetrahedral grid

Repaired mesh surface

Mesh from

surface to volume

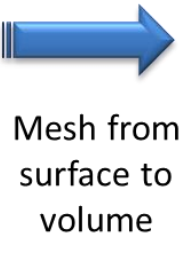

Figure 4 Schematic of FE mesh process

For the tensile test, the FEM model based on the tomographic image with a voxel size of $4.5 \mu \mathrm{m}$ was used. The material property was set as elastoplastic, the stress-strain curve deduced from a conventionally standard test on A319 LFC alloy (Figure 3(b)) was used to define the yield strength, Young modulus and strain hardening law for FEM simulation. The loading condition was an imposed uniform displacement on the upper and lower boundaries of the FEM model. The displacement value was chosen to obtain a macroscopic deformation along the loading direction in the FEM model that is about the same as the average deformation measured in the CT image during the in-situ test at the highest load.

For fatigue tests, the FE mesh model was extracted from the SR-CT reference image at the unloaded state. The same ROI was used for FEM simulation and DVC, i.e. about $2.6 \mathrm{~mm}$ along the specimen gauge length. The material property is the same as the one used for the tensile test on A319 LFC alloy (Figure 3(b)). FEM simulation was performed in cyclic condition using nonlinear isotropic/kinematic hardening model identified from experimental cyclic stress-strain loops. The average maximum strain at $1^{\text {st }}$ cycle deduced from DVC was used to compute the imposed displacement on the FE model boundaries. The equivalent plastic strain at integration points (PEEQ), which measures the amount of permanent strain in material, was computed. It is the total accumulation of plastic strain and is defined as $P=\int_{\mathrm{t}} \sqrt{\frac{2}{3} d \varepsilon^{p l}: d \varepsilon^{p l}} d t$, where $d \varepsilon^{p l}$ is the increment plastic strain, while $\mathrm{t}$ stands for the loading history from the first cycle to the current calculated cycle. 


\section{Results and discussion}

\subsection{Selection of ROI and representative specimens}

An example of 3D rendering of pores in the bulk of one small specimen selected for tensile tests is shown in Figure 5(a). Numerous large pores were found in the central part of the specimen and some of them having sharp shape are expected to produce large stress concentrations. Thus the zone in the neighborhood of large pores was selected as ROI.

The distributions of pores in the selected specimen (analyzed volume is about $8.2 \mathrm{~mm}^{3}$ ) and in a larger volume (analyzed volume is about $70 \mathrm{~mm}^{3}$ ) were compared in Figure 5(b). The green (resp. red) curve and histogram stand for the pores distributions in the specimen (resp. studied material) with a voxel size of $4.5 \mu \mathrm{m}$ (resp. $3 \mu \mathrm{m}$ ) in number and volume frequency, respectively. The first peak in the number distribution corresponds to small rounded gas pores: the small difference between the test specimen and the material may be caused both by the different resolutions used, which controls the detectability of small pores, and by the scatter in microstructure. The peak in the volume distribution corresponds to large microshrinkage cavities, which are fewer in number but do represent most of the pores volume fraction. Besides, in the specimen, the volume fraction of porosity is $0.88 \%$, which is close to that measured in the studied material ( 1\%), and the maximum size of pores is about $1.05 \mathrm{~mm}$, which is a typical size for large defects in this material. This largest pore is a subsurface shrinkage pore. Even small size specimen may thus be representative of the studied material in terms of the size distribution of small pores and most importantly of large pores, which correspond to the peak in the volume distribution in Figure 5(b), that are believed to play the most important part in damage initiation. The majority of pores, i.e. pores occupying more than $90 \%$ volume of total pores, have a Feret diameter larger than $0.4 \mathrm{~mm}$.

(a)

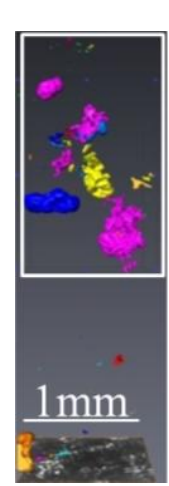

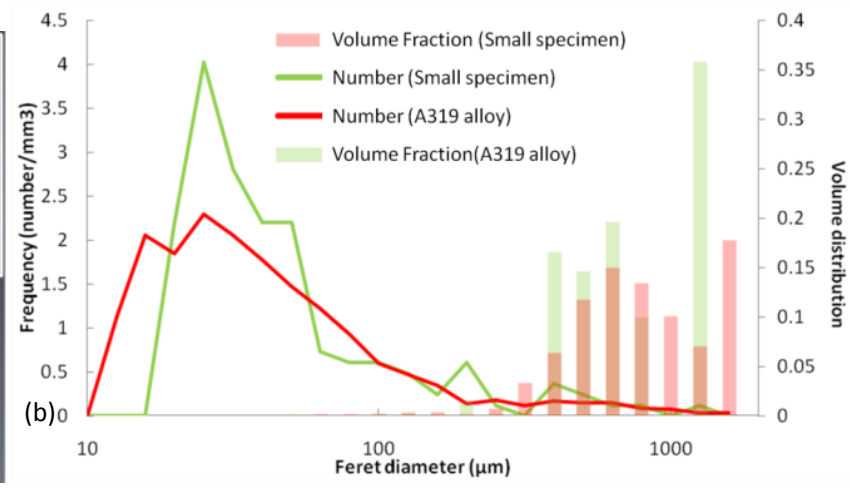

Figure 5 (a) 3D rendering of pores in the selected tensile specimen (Zone of interest is delimited by a rectangle box) and (b) size distributions of pores for small (tensile) and large specimens 


\subsection{Extraction of $3 D$ cracks using DVC}

Figure 6 shows the displacement fields and strain fields measured from DVC for the tensile test at different loading steps. No obvious cracks were observed at the first step (147MPa) in the tomography image, and both the displacement and strain fields are uniform. Cracks initiations were observed at the third step (176MPa) in the area marked with an arrow in the displacement field. Then displacement discontinuities and localizations in the strain field, where cracks nucleated and propagated, were observed to increase at increasing load.
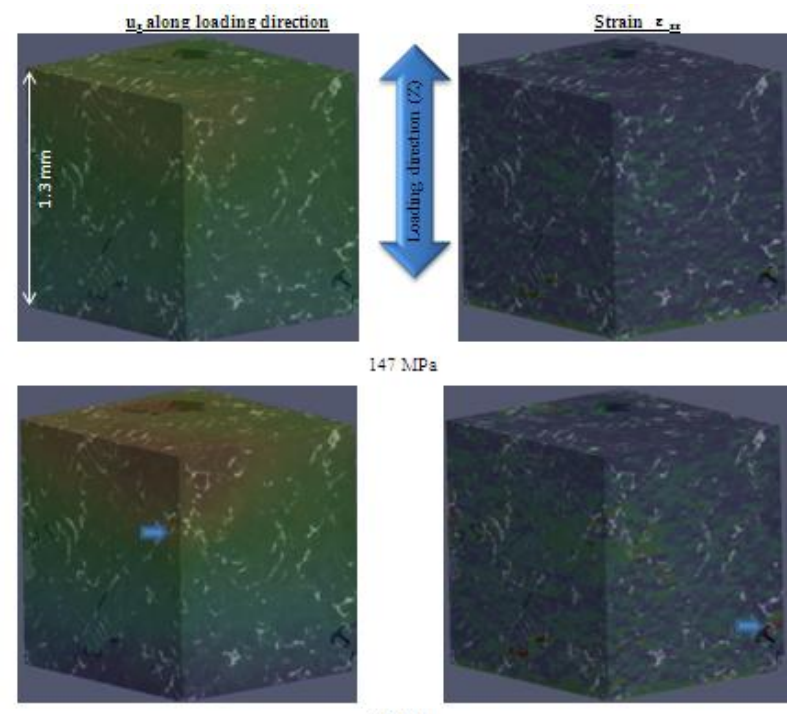

$147 \mathrm{MPa}$
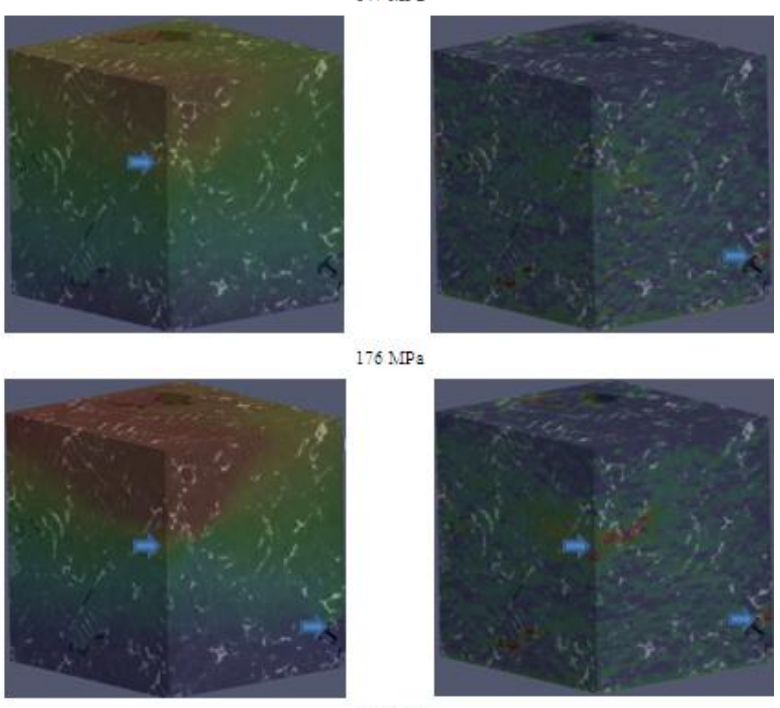

$176 \mathrm{MPa}$
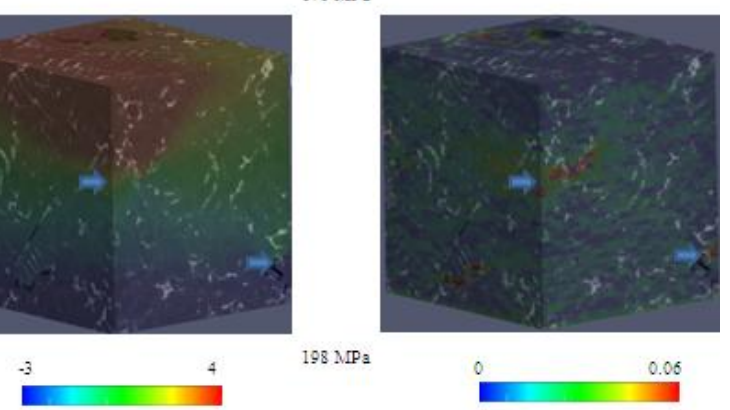

Figure 6 Displacement fields (in pixels, 1 pixel $=1.695 \mu \mathrm{m}$ ) and strain fields of tensile specimen

The displacement discontinuity induced by the crack avoids matching the reference image to the image corrected by the measured displacement field. This so-called residual error, which could be calculated as 'Reference image + calculated displacement fields - deformed image', is maximum at the cracks locations and gives information about the crack geometry (Figure 7). The thresholding of the original tomographic image is difficult due to the limited opening of the crack compared to the voxel size. Thus the residual error provides a less arbitrary way to extract the cracks than greyscale thresholding from the tomography image ${ }^{[24]}$. 3D cracks in the following analysis of tensile tests were extracted using the maximum residual error. 


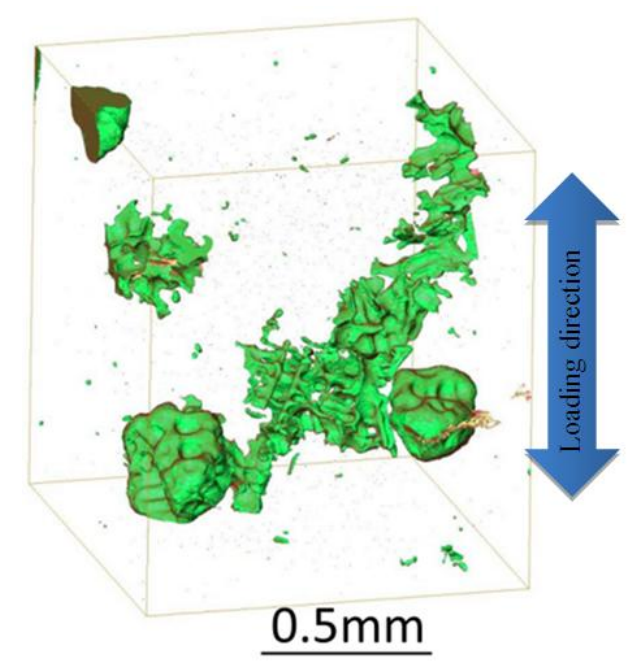

Figure 7 comparison of residual error in yellow and cracks segmented (roughly) in red color

However the above extraction of 3D cracks does not work in 3D fatigue tests although the relation between cracks or final fracture and displacement discontinuities was also observed ${ }^{[25]}$. The values of residual error in some areas along the 'white colour hard inclusions' (copper containing phases and some iron intermetallics) in the tomographic image are the same as in the cracks. Thus it was impossible to extract 3D cracks from the maximum residual errors. This problem was mainly caused by the too strong phase contrast provided by SR-CT. For example, the interfaces of copper containing phase might present different grey levels in the constructed images at different steps due to the reconstruction errors of phase contrast imaging. Such a local brightness variation at this phase yielded large residual error around this phase. Fortunately, the phase contrast influence was not observed on displacement or strain field. The smaller opening of cracks in fatigue tests than in tensile tests is also one possible reason. In view of the above arguments, the 3D cracks in fatigue tests could only be extracted from the direct thresholding of the tomographic images, although this is a tedious work which had to be carried out semi automatically.

\subsection{Crack initiation}

Tensile test: The cracks growth with the load increasing from $176 \mathrm{MPa}$ to $198 \mathrm{MPa}$ is shown in Figure 8 . The observation direction for these three images is along the loading direction. Pores are shown in translucent grey and cracks in translucent yellow. These 3D renderings of cracks and pores illustrate the process of crack initiation and growth during tensile test. The cracks nucleated from large surface and subsurface pores at $176 \mathrm{MPa}$ (Figure 8(a)). Then they grew with a further increase in the applied stress (Figure $8(\mathrm{~b})(\mathrm{c})$ ). When cracks initiated from a subsurface pore, they did so from the ligament between the pore and the free surface and further propagated towards the free surface. 

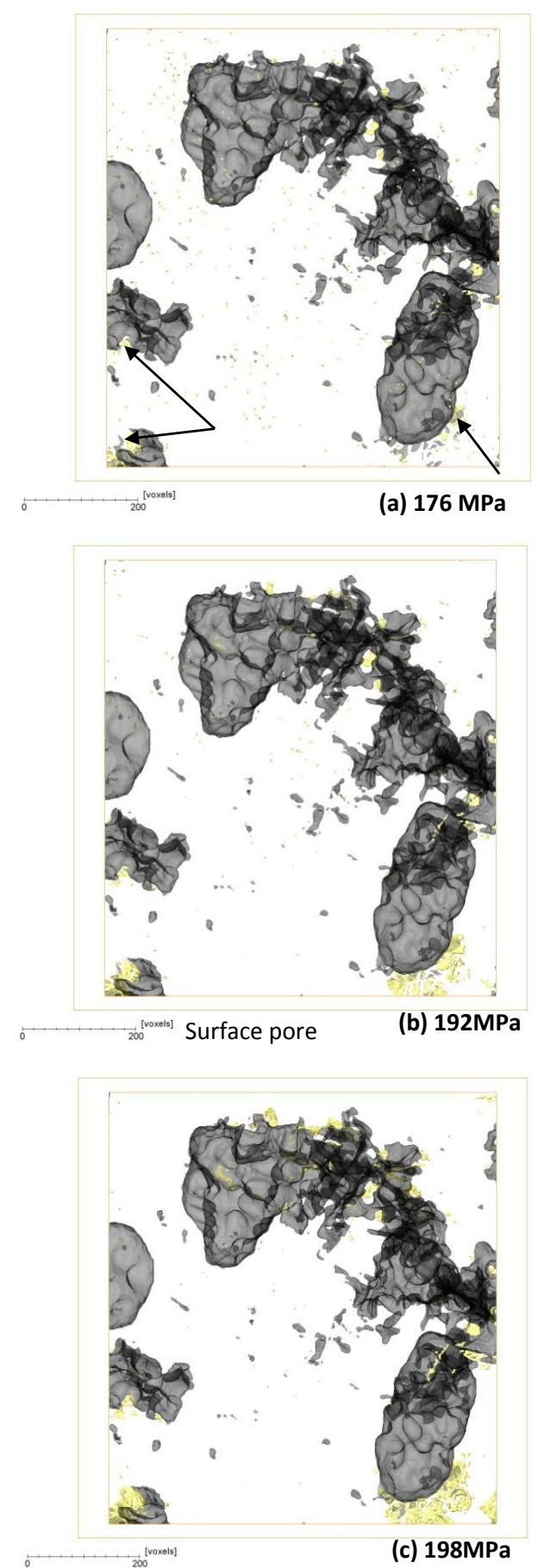

Figure 8 Cracks nucleation and growth around pores at increasing load during the tensile test (Pores in translucent grey and cracks in translucent yellow)

Fatigue test: One fatigue test is presented in this paper. The maximum applied stress was about $130 \mathrm{MPa}$, and the average maximum longitudinal strain deduced from DVC was about $0.14 \%$. The last scan was taken at the $10500^{\text {th }}$ cycle, just a few cycles before the specimen's failure. One large crack was observed around the largest pore in the specimen (see Figure 9). In fact this large crack initiated during the load increase up to the maximum load of $1^{\text {st }}$ cycle $(1 / 2$ cycle) from the surface of the largest pore. All the fatigue tests in this study were realized with 
maximum stresses lower than the stress level at which cracks initiations were observed in the previous tensile tests with 3D in-situ observations (i.e. $176 \mathrm{MPa}$ ). For all the fatigue tests, cracks initiations were always observed during the tensile stage of $1^{\text {st }}$ cycle. Comparisons between Figure 9 (a) and (b) show that the displacement discontinuities are observed at crack and final fracture locations and become more obvious with cyclic loadings.

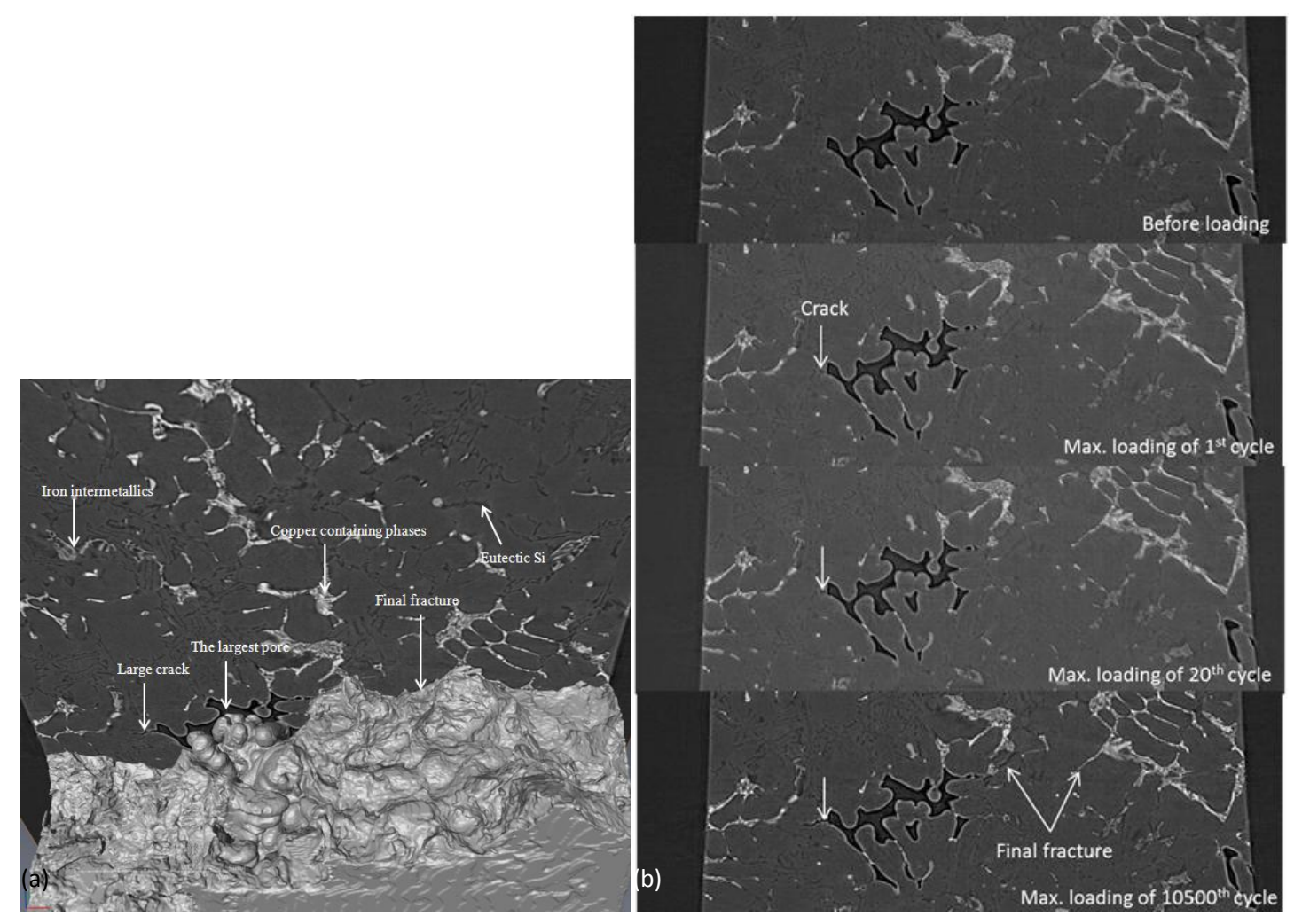

Figure 9 (a) One slice (about $680 \mu \mathrm{m}$ below the fatigue surface) of SR-CT image taken at the maximum loading of $10500^{\text {th }}$ cycle shown with 3D rendered fracture surface; (b) SR-CT images of the slice shown in (a) at different cycles

In both tensile and LCF tests, cracks initiated from large pores' surfaces upon monotonic tensile loading. During he tensile tests, the large stress concentration around large pores resulted in crack initiation between 165 and 176MPa. In LCF, even in the first $1 / 2$ cycle, the stress concentration around the large pore present in the sample gauge length was also large enough to induce crack initiation. Thus in the LFC A319 alloy in both tensile and cyclic loading: large pores due to LFC produce large stress concentrations which induce crack initiation at the pores' sharp corners.

The location of the pore is also important. The cracks in the tensile specimen initiated from large subsurface and surface pores at 176MPa. Even if the surface pores are smaller than some other pores, many cracks initiated from them (Figure 8). This is consistent with [17, 18]: Surface pores are prone to act as crack initiation sites as they create regions of higher stress concentrations. Several cracks also initiated from the largest pore, which is a subsurface pore 
near the free surfaces where a higher stress concentration ${ }^{[32]}$ is achieved.

\subsection{Comparisons between experimental and numerical fields}

Cracks initiated from large pores' surfaces where strain localizations were measured. Thus, in order to better understand the influence of pores on strain heterogeneities and to discriminate it from the influence of other microstructural constituents, numerical fields using 3D FE model based on pores and solid matrix were compared with experimental fields deduced from DVC.

The strain field from the FEM simulation of the tensile test is shown in Figure 10(a). The sub volume where the DVC measurement was performed is marked with a red rectangle in Figure 10(a) and is shown at a higher magnification in Figure 10(b) in order to compare with the strain field at 198MPa from DVC in Figure 10(c). The similarity between the strain fields from FEM and from DVC confirms that the strain localization observed in the experimental fields can be mostly ascribed to pores and that hard particles have a less important influence.

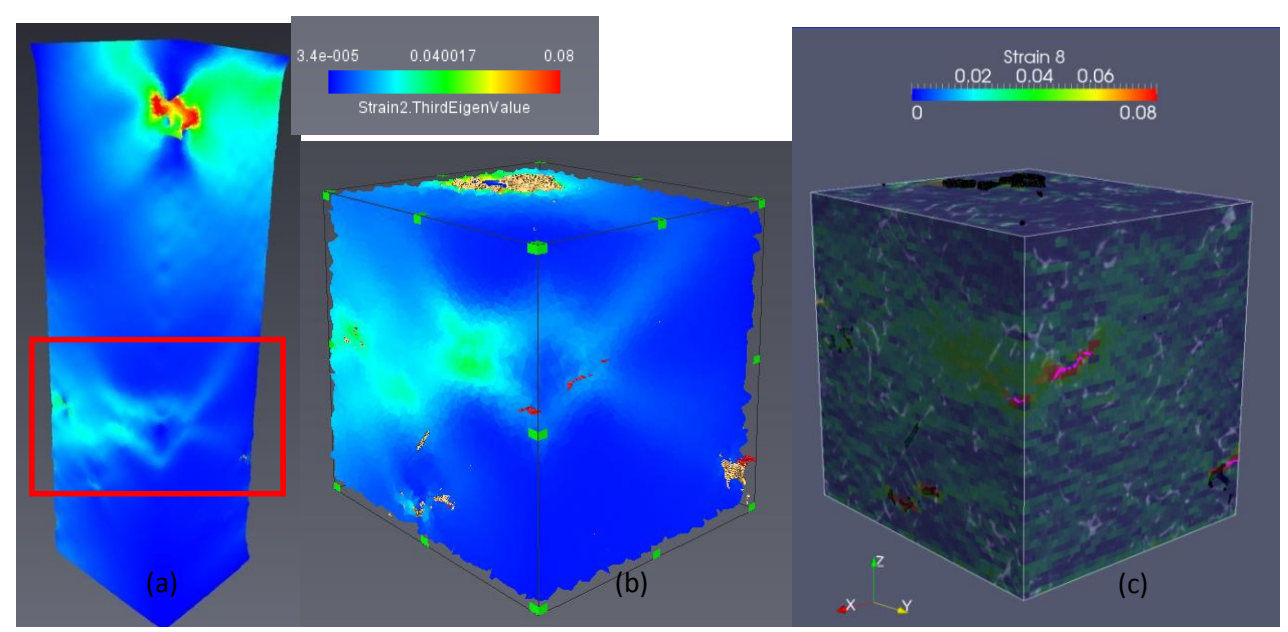

Figure 10 Comparison between FE simulation and DVC measurements for tensile test: (a) Strain field ( $\varepsilon z z)$ from FEM with (b) a focus on the sub volume in the area marked with a red rectangle in (a) that corresponds to the area where the DVC measurements were performed (pores shown in golden, cracks in red) and corresponding strain field from DVC at 198MPa (pores shown in black and cracks in purple)

A comparison between FE simulation and DVC measurements for the fatigue test is shown in Figure 11. Strain localizations occur around the largest pore (maximum Feret diameter $=1.22$ $\mathrm{mm}$ ) (Figure 11(c)). The cracks (Figure 9) initiated at the highest strain localizations which are caused by local sharp shapes. 


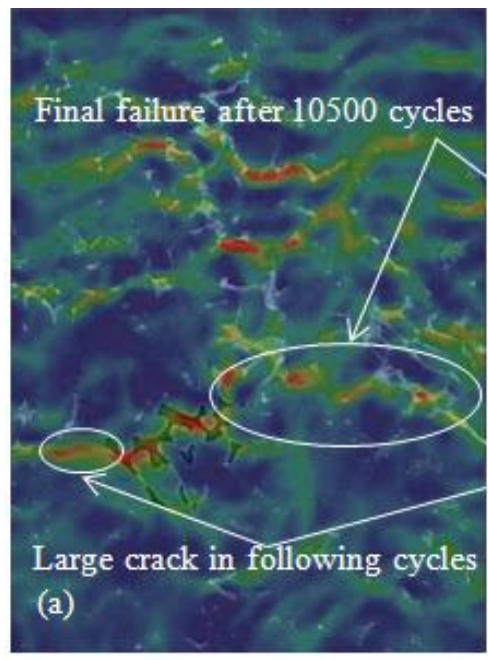

0

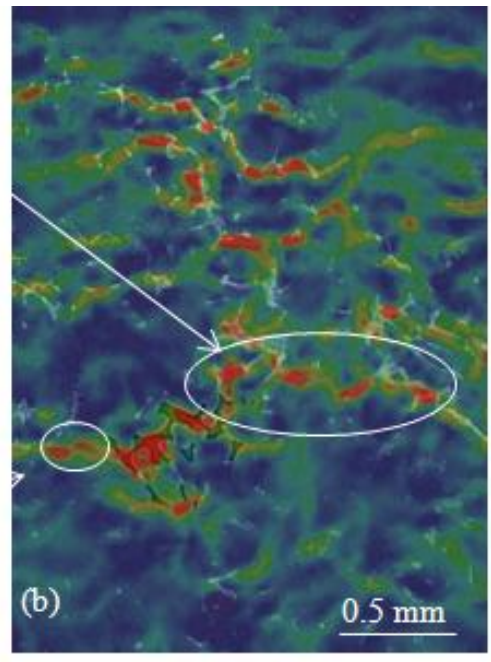

0.01

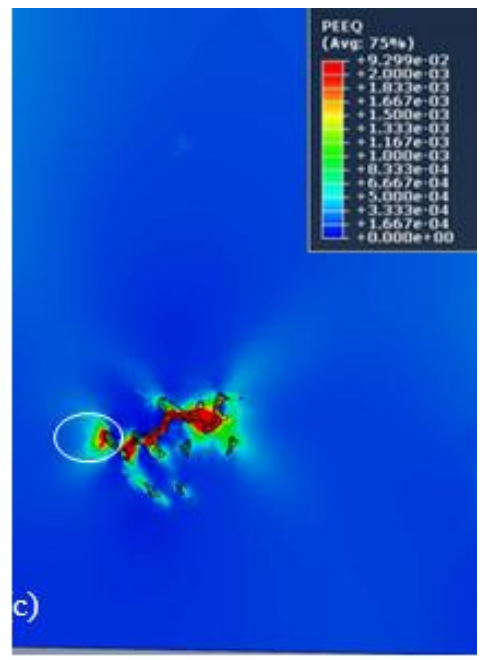

Figure 11 Comparison between FE simulation and DVC measurements for fatigue test: Equivalent Von Mises strain field from DVC: correlation between the minimum loading of $1^{\text {st }}$ cycle and the maximum loading of (a) $2^{\text {nd }}$ cycle and (b) 20th cycle. (c) PEEQ field (after 3 cycles) from FE simulation. (Slice shown is about $680 \mu \mathrm{m}$ below the flat surface)

The DVC results obtained for the fatigue tests (i.e. element size $=13 \mu \mathrm{m}$ ) have a higher spatial resolution than that for tensile tests (i.e. element size $=27.2 \mu \mathrm{m}$ ), thus the former can show finer strain localizations than the later. Strain localizations were only observed at pores in DVC fields of tensile tests as well as in FEM fields. However, in DVC fields of fatigue tests (Figure 11 (a) and (b)), strain localizations are also observed at several scales: macroscopic (compared with the small size of specimen) strain localizations at pores, which were also observed in FEM fields (Figure 11 (c)), and strain localizations at hard inclusions near pores which could not be observed in the computed FEM fields (Figure 11 (c)) but were observed to rapidly increase with cyclic loadings in the measured DVC fields (Figure 11 (a) and (b)). In the slice shown in Figure 9 and in Figure 11, the main crack was still very small at 20 cycles and the displacement discontinuity is hardly visible in Figure 9(b) so that opening of crack almost has no influence on strain localization in the mechanical field measured by DVC. Therefore, the strain localization observed in Figure 11(a) and (b) on each side of the pore is mainly due to the strain concentration at the pore itself as proved by the comparison between DVC and FEM. Besides the main crack and final fracture area, it is impossible to know if there are microcracks at other strain localizations areas until final failure due to the limited resolution of tomographic images. However strain localization is also observed on apparently crack free areas at hard inclusions (see arrow in Figure 11(b)). The propagation of large cracks and final failure went through these areas where strain localizations are observed at hard inclusions. This indicated that even if pores have the most important influence on strain localizations for crack initiation, hard inclusions may play an important role for strain distribution affecting crack propagation and final fracture. 


\section{Conclusions}

In order to study the influence of the microstructure of LFC A319 alloy upon its mechanical behavior, an experimental protocol has been set up using X-ray tomography and DVC. The pore distributions were firstly characterized in 3D. Afterwards, the protocol was validated during tensile tests and was then applied to a fatigue test.

(1) The Feret diameter has been used to characterize both the size and shape distribution of pores, while the "sphericity" was used to distinguish between gas pores and shrinkage pores.

(2) The correlation residual error, which is maximum at the cracks locations, can be used to extract the cracks in the absence of phase contrast (Lab CT).

(3) The DVC measurement on LFC A319 alloy can be considered reliable based on uncertainty measurements and on the comparison between the stress-strain curve derived from DVC and an experimental curve obtained with conventional extensometry on a macroscopic sample.

(4) Cracks were observed to initiate at large pores and microshrinkage cavities both in monotonic tensile and cyclic loadings.

(5) Pore has an important influence on strain localization and large pores generate enough strain concentration zones which are large enough for crack initiation to occur during the first loading cycle in LCF. This is confirmed by FEM.

(6) Hard inclusions play important role for strain distribution affecting crack propagation and final fracture in cyclic loadings.

The analysis of fatigue tests are currently being performed and focus on the stage of crack propagation as well as the influence of hard inclusions. This developed experimental protocol is also being applied to study the damage micromechanisms of LFC A319 alloy at high temperature $\left(200-250^{\circ} \mathrm{C}\right)$.

\section{Acknowledgements}

This research work was funded by INDiANA-ANR project (grant ANR-12-RMNP-0011) and PSA Peugeot Citroën. The authors would like to thank TOMCAT beamline at Swiss Light Source (SLS) for providing SR-CT beamtime, the China Scholarship Council (SLS) for funding the PhD thesis of Long WANG and Julien Réthoré (LaMCoS laboratory) for the use of MIC3D. 


\section{References}

1 Gruzleski, J.E., Closset, B.M., and Society, A.F. (1990). The treatment of liquid aluminum-silicon alloys (American Foundrymen's Society, Inc.).

2 Hirsch, J. (2011). Aluminium in Innovative Light-Weight Car Design.Mater. Trans. 52, 818-824.

3 Wang, Q.G., Apelian, D., and Lados, D.A. (2001). Fatigue behavior of A356-T6 aluminum cast alloys. Part I. Effect of casting defects. J. Light Met. 1, 73-84.

4 Wang, Q.G., Apelian, D., and Lados, D.A. (2001). Fatigue behavior of A356/357 aluminum cast alloys. Part II - Effect of microstructural constituents. J. Light Met. 1, 85-97.

5 Rajput, R.K. (2007). A Text Book of Automobile Engineering (Firewall Media).

6 Niane, N.T., and Michalet, J.-P. (2011). Validation of Foundry Process for Aluminum Parts with Flow3D Software. In Proceedings of the 2011 International Symposium on Liquid Metal Processing and Casting, 2011, (New York),.

7 Geffroy, P.-M., Lakehal, M., Goñi, J., Beaugnon, E., Heintz, J.-M., and Silvain, J.-F. (2006). Thermal and mechanical behavior of Al-Si alloy cast using magnetic molding and lost foam processes. Metall. Mater. Trans. A 37, 441-447.

8 González, R., Martínez, D.I., González, J.A., Talamantes, J., Valtierra, S., and Colás, R. (2011). Experimental investigation for fatigue strength of a cast aluminium alloy. Int. J. Fatigue 33, 273-278.

9 Dobrzański, L.A., Borek, W., and Maniara, R. (2006). Influence of the crystallization condition on Al-Si-Cu casting alloys structure. J. Achiev. Mater. Manuf. Eng. 18, 211-214.

10 Tabibian, S. (2011). Contributions to Thermomechanical Fatigue Criteria for Lost Foam Casting Aluminum Alloys: Ph.D thesis. Ecole Centrale de Lille.

11 Wang, Q.G., and Cáceres, C.H. (1997).Mg Effects on the Eutectic Structure and Tensile Properties of Al-Si-Mg Alloys.Mater. Sci. Forum 242, 159-164.

12 Kaufman, J.G., and Rooy, E.L. (2004). Aluminum Alloy Castings: Properties, Processes, and Applications (ASM International).

13 Boileau, J.M., and Allison, J.E. (2003).The effect of solidification time and heat treatment on the fatigue properties of a cast 319 aluminum alloy. Metall. Mater. Trans. A 34, 1807-1820.

14 Gall, K., Horstemeyer, M.F., Degner, B.W., McDowell, D.L., and Fan, J. (2001).On the driving force for fatigue crack formation from inclusions and voids in a cast A356 aluminum alloy. Int. J. Fract. 108, 207-233.

15 Mu, P., Nadot, Y., Nadot-Martin, C., Chabod, A., Serrano-Munoz, I., and Verdu, C. (2014). Influence of casting defects on the fatigue behavior of cast aluminum AS7G06-T6. Int. J. Fatigue 63, 97-109.

16 Zhang, H., Toda, H., Hara, H., Kobayashi, M., Kobayashi, T., Sugiyama, D., Kuroda, N., and Uesugi, K. (2007).Three-Dimensional Visualization of the Interaction between Fatigue Crack and Micropores in an Aluminum Alloy Using Synchrotron X-Ray Microtomography. Metall. Mater. Trans. A 38, 1774-1785. 
17 Ammar, H.R., Samuel, A.M., and Samuel, F.H. (2008).Porosity and the fatigue behavior of hypoeutectic and hypereutectic aluminum-silicon casting alloys. Int. J. Fatigue 30, 1024-1035.

18 Gao, Y.X., Yi, J.Z., Lee, P.D., and Lindley, T.C. (2004). A micro-cell model of the effect of microstructure and defects on fatigue resistance in cast aluminum alloys. Acta Mater. 52, $5435-5449$.

19 Buffière, J.-Y., Savelli, S., Jouneau, P.H., Maire, E., and Fougères, R. (2001). Experimental study of porosity and its relation to fatigue mechanisms of model $\mathrm{Al}-\mathrm{Si} 7-\mathrm{Mg} 0.3$ cast $\mathrm{Al}$ alloys. Mater.Sci. Eng. A 316, 115-126.

20 Nicoletto, G., Konečná, R., and Fintova, S. (2012). Characterization of microshrinkage casting defects of Al-Si alloys by X-ray computed tomography and metallography. Int. J. Fatigue 41, 39-46.

21 Stolarz, J., Madelaine-Dupuich, O., and Magnin, T. (2001). Microstructural factors of low cycle fatigue damage in two phase Al-Si alloys. Mater.Sci. Eng. A 299, 275-286.

22 Salvo, L., Suéry, M., Marmottant, A., Limodin, N., and Bernard, D. (2010).3D imaging in material science: Application of X-ray tomography. ComptesRendus Phys. 11, 641-649.

23 Limodin, N., Réthoré, J., Buffière, J.-Y., Gravouil, A., Hild, F., and Roux, S. (2009). Crack closure and stress intensity factor measurements in nodular graphite cast iron using three-dimensional correlation of laboratory X-ray microtomography images. Acta Mater.57, 4090-4101.

24 Limodin, N., Réthoré, J., Buffière, J.-Y., Hild, F., Roux, S., Ludwig, W., Rannou, J., and Gravouil, A. (2010). Influence of closure on the 3D propagation of fatigue cracks in a nodular cast iron investigated by X-ray tomography and 3D volume correlation. Acta Mater.58, 2957-2967.

25 Wang L. (2015). Influence of the casting microstructure on damage mechanisms in Al-Si alloys by using 2D and 3D in-situ analysis: Ph.D thesis. Ecole Centrale de Lille.

26 Buffiere, J.-Y., Maire, E., Adrien, J., Masse, J.-P., and Boller, E. (2010). In Situ Experiments with $\mathrm{X}$ ray Tomography: an Attractive Tool for Experimental Mechanics. Exp. Mech. 50, 289-305.

27 Lachambre, J. (2014). Développement d'une Méthode de Caractérisation 3D des Fissures de Fatigue à l'aide de la Corrélation d'Images Numériques obtenues par Tomographie X. Ph. D. thesis, Institut National des Sciences Appliquées de Lyon.

28 Sutton, M.A., Orteu, J.J., and Schreier, H. (2009). Image Correlation for Shape, Motion and Deformation Measurements: Basic Concepts, Theory and Applications (New York: Springer).

29 Seghir, R., Witz, J.F. and Coudert, S., YaDICs - Digital Image Correlation 2/3D software, http://www.yadics.univ-lille1.fr, 2014.

30 Limodin, N., El Bartali, A., Wang, L., Lachambre, J., Buffiere, J.-Y., and Charkaluk, E. (2014).Application of X-ray microtomography to study the influence of the casting microstructure upon the tensile behaviour of an Al-Si alloy.Nucl.Instrum. Methods Phys. Res. Sect. B Beam Interact. Mater.At.324, 57-62.

31 ZIB (Konrad-Zuse-ZentrumfürInformationstechnik Berlin), and VSG (Visualization Sciences Group).(2012). Avizo Online Help http://www.fei.com/software/avizo-3d-resources/.

32 Fan, J., McDowell, D.L., Horstemeyer, M.F., and Gall, K. (2003). Cyclic plasticity at pores and inclusions in cast Al-Si alloys. Eng. Fract. Mech. 70, 1281-1302. 